CUADERNOS DE ESTUDIOS GALLEGOS, LXI Núm. 127 (enero-diciembre 2014), págs. 299-317

ISSN: $0210-847 \mathrm{X}$

DOI: 10.3989/ceg.2014.127.10

\title{
EL «COMPENDIO DE EXPLORACIÓN MÉDICA» DE NÓVOA SANTOS Y VILA BARBERÁ
}

\author{
Fernando J. Ponte Hernando* \\ Servicio Galego de Saúde \\ José Pascual Bueno; Ana Pascual Molina \\ Universidad Católica de Valencia \\ Sonia GonzÁlez Castroagudín \\ Universidad de A Coruña
}

* Grupo de Investigación GI-1565 OARMA (USC) 


\section{EL «COMPENDIO DE EXPLORACIÓN MÉDICA» DE NÓVOA SANTOS Y VILA BARBERÁ}

RESUMEN

En la Edad de Plata de la Cultura española (1898-1936), la medicina no fue ajena al nuevo Renacimiento. A ello contribuyó la aparición de una serie de figuras, nacidas a partir de 1870; la llegada de la moderna medicina científica de Francia, con la obra de Magendie, Claude Bernard, Luis Pasteur, y de Alemania con Virchow, Koch, von Krehl y otros; así como la salida de nuestros médicos a formarse allí, gracias a la actividad de la JAE fundada en 1907, durante el reinado de Alfonso XIII, y presidida por D. Santiago Ramón y Cajal.

Por primera vez, desde el Siglo de Oro, la medicina española estaba entre las mejores. Junto a las traducciones de obras extranjeras, surge una estimable producción propia. La obra neurohistológica de Cajal y Tello, con sus correspondientes clínicos en Achúcarro, Simarro, etc. y la aparición de tratados de medicina como el de Hernando y Marañón, la Patología General de Nóvoa Santos y el monumental Tratado Iberoamericano de Medicina Interna de Fidel Fernández, son una muestra de ello.

Sin embargo, la medicina científica a la cabecera del enfermo demandaba alguna obra de exploración clínica completa pero asequible. Existía ya la obra de Noguer Molins, que data de 1916, pero no incluía radiología y laboratorio.

Presentamos el primer intento, inédito hasta ahora, de edición de un manual completo y asequible de exploración clínica, de radiología y laboratorio, llevado a cabo por dos de las figuras de la medicina de la época: los catedráticos de Patología General de Madrid y Valencia, Roberto Nóvoa Santos y Ramón Vila Barberá.

Palabras Clave: Nóvoa Santos, Vila Barberá, Exploración médica, Semiología, Compendio, Manual.

\section{O «COMPENDIO DE EXPLORACIÓN MÉDICA» DE NÓVOA SANTOS E VILA BARBERA}

RESUMO

Na Era de Prata da cultura española (1898-1936), a medicina non era inmune ao novo Renacemento. Isto contribuíu a aparición dunha serie de figuras, nacidas a partires de 1870; coa chegada da moderna medicina científica en Francia, co traballo de Magendie, Bernard, Pasteur, e Alemaña, con Virchow, Koch, von Krehl e outros; e a saída dos nosos médicos para formar alí, grazas á actividade da JAE fundada en 1907, durante o reinado de Alfonso XIII e presidido por D. Santiago Ramón y Cajal.

Por primeira vez dende a Idade de Ouro da medicina española estaba entre os mellores. Xunto con traducións de obras estranxeiras, ven unha producción propia estimáveis. O traballo neuro-histolóxico de Cajal e Tello, co seu correspondente clínico en Achúcarro, Simarro, etc. e a aparición de tratados médicos como o de Hernando e Marañón, a Patoloxía Xeneral de Nóvoa Santos e o monumental Tratado Iberoamericano de Medicina Interna de Fidel Fernández, son un exemplo diso. Con todo, a medicina científica á cabeceira esixía un tratado de exame clínico completo pero accesible. Xa o foi o traballo de Noguer Molins, que data de 1916, pero non incluíu radioloxía e laboratorio.

Presentamos o primeiro intento, ata agora inédito, a edición dun manual de exame clínico completo e accesible, con radioloxía e laboratorio, realizada por dúas das figuras en medicina da época: os profesores de Patoloxía Xeral de Madrid e Valencia Roberto Novoa Santos e Ramón Vila Barbera.

Palabras Clave: Nóvoa Santos, Vila Barberá, Exame médico, Semioloxía, Compendio, Manual.

\section{«CLINICAL EXAMINATION TREATISE» BY NÓVOA SANTOS AND VILA BARBERÁ}

Abstract

During the Spanish culture Silver Age (1898-1936), medicine was not indifferent to this new Reinaissance. And it was owing to the birth of some important Spanish figures, born since 1870; to the arrival of the modern scientific medicine from France with Magendie, Bernard, Pasteur's works, and from Germany with Virchow, Koch, von Krehl and others' works; and to the fact that our doctors went there to study, thanks to the activity of the JAE., founded in 1907, during Alfonso XIII's reign, and whose president was D. Santiago Ramón y Cajal.

For the first time since the Golden Age, Spanish medicine was among the best in the world. Next to translations of foreign works, a valuable own output comes up. Cajal and Tello's neurohistological work, with Achúcarro, Simarro, etc. and the appearance of medicine treatises like that one written by Hernando and Marañón, Nóvoa Santos' General Pathology and the monumental Ibero-American treatise on Internal Medicine by Fidel Fernández, are a good example of this.

However, scientific medicine for general practitioners was asking for a complete but feasible clinical examination work. Noguer Molins had written one in 1916, but it did not include radiology or laboratory

We are presenting the first attempt, unknown up to now, of publishing a complete feasible manual on clinical examination, radiology and laboratory, written by two of the most important medicine figures in that time: General Pathology chairs in Madrid and Valencia, Roberto Nóvoa Santos and Ramón Vila Barberá.

KeY WoRDS: Nóvoa Santos, Vila Barberá, Physical examination, Semiology, Treatise, Handbook. 
Recibido/Received: 12/05/2014

Aceptado/Accepted: 23/09/2014

finales de los años 20 del pasado siglo, los manuales de exploración clí-
nica más usados en España eran el Seifert y Muller, que, en 1887, ya iba
por su tercera edición ${ }^{1}$ y había sido multieditado desde finales del XIX; y el de D. Luis Noguer Molins ${ }^{2}$, obra nacida en 1916, que bajo las diferentes supervisiones del Prof. Balcells Gorina y recientemente, desde hace unos años, del Prof. Prieto Valtueña, ha llegado hasta nuestros días. No obstante parece ser que, los autores de los que hablaremos a continuación, Nóvoa Santos y Vila Barberá (Fig. 1), querían que su compendio fuese más breve que el primero y más amplio que el segundo, incluyendo capítulos de radiología y laboratorio.

\section{El Compendio NóvoA-Vila}

Por el hallazgo de una $\operatorname{carta}^{3}$ (Fig. 2 y Fig. 3) dirigida al Prof. Dr. D. Miguel Gil Casares, Catedrático de Patología y Clínica Médicas de la Universidad de Santiago, que podemos datar hacia verano de 1929, hemos sabido que, en esa época, los catedráticos de Patología General de Madrid y Valencia, D. Roberto Nóvoa Santos y D. Ramón Vila Barberá ${ }^{4}$, llegaron a la conclusión de que se hacía preciso desarrollar lo que llamaron:

\footnotetext{
1 Otto SeIfert y Friedrich MüLler, Guía del diagnóstico médico, Madrid, Tip. de Enrique Teodoro, 1887 .

2 Luis Noguer Molins, distinguido maestro catalán que, bien entrados los años 40, se exilió a Venezuela y había leído su tesis doctoral sobre la Linitis plástica, en 1913 en la Universidad Central de Madrid. De vuelta en España, el 18 de mayo de 1961, fue objeto de un homenaje institucional en Barcelona por los 45 años de su libro, y de su ejemplar magisterio. Vid. Periodico La VANGUARDIA [en línea], disponible en <http://hemeroteca.lavanguardia.com/preview/1961/05/19/pagina-19/32701756/pdf.html> [Consulta: 30/04/2014].

3 La carta que da origen a este artículo la debemos a la generosidad de la familia de D. Miguel Gil Casares.

4 Ramón Vila Barberá (1880-1941). Estudió Medicina en Valencia y se doctoró en Madrid en 1904 con la tesis: Tratamiento de las enfermedades del corazón por el ejercicio y la gimnástica. Entró como profesor auxiliar de Patología Médica por oposición a la Facultad de Medicina de Valencia en 1910. En 1921 consigue la cátedra de Higiene y Bacteriología en Salamanca; regresa a Valencia en 1923 ocupando la cátedra de Patología General. Además fue académico numerario de la Real Academia de Medicina de Valencia, ingresando el 21 de febrero de 1926 con el discurso: Algunas
} 

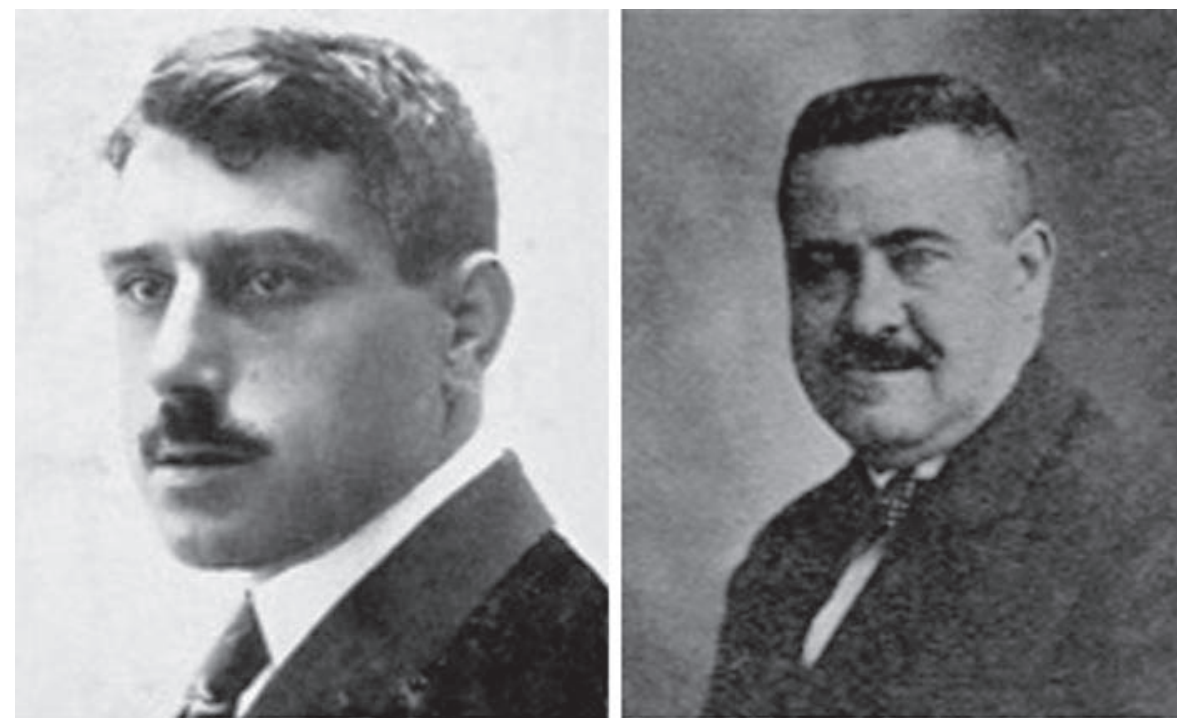

Fig. 1

Un compendio de exploración clínica y de laboratorio clínico que, con claridad y concisión, exponga al día cuanto al práctico y al estudiante interesan en estas materias, de tal modo, que con sólo esta Guía pueda aplicar en la clínica y en el laboratorio sus técnicas exploratorias con finalidad diagnóstica y pronóstica.

Para ello, tras encargar papel con membrete de ambos, en Madrid y Valencia, como vemos en la figura 2, elaboran un programa de dicho proyecto editorial al que llaman Compendio de Exploración Médica (Clínica y Laboratorio) que dividen en tres grandes apartados que, asimismo, subdividen en otros tres, con sus correspondientes directores:

adquisiciones recientes en metabolimetría clínica, que fue contestado por D. Vicente Peset Cervera. En la misma corporación pronunció el discurso de apertura de curso de 1931 sobre: "El prerriñón y el riñón en el metabolismo del agua". Fundó la revista mensual Policlínica, excelente publicación que duró de 1913 a 1933, y escribió numerosos artículos de divulgación científica. Fue vocal de la Junta directiva de la Sociedad Española de Hidrología Médica. Al frustrarse este proyecto publicó en 4 tomos y un $5^{\circ}$ que quedó inédito por su muerte el tratado denominado: Medicina Exploratoria: Clínica y Laboratorio, entre 1932 y 1941, año de su fallecimiento en el balneario de Liérganes (Santander). Rafael Campos Fillol, Crónica de la Facultad de Medicina de Valencia (De 1866 a 1946), Discurso de recepción del académico electo Prof. Rafael Campos Fillol, Valencia, Real Academia de Medicina de Valencia, 1955, y José María López Piñero, Historia de la Medicina Valenciana, tomo III, Valencia, Ed. Vicent García, 1992, págs. 112, 116 y 117. 


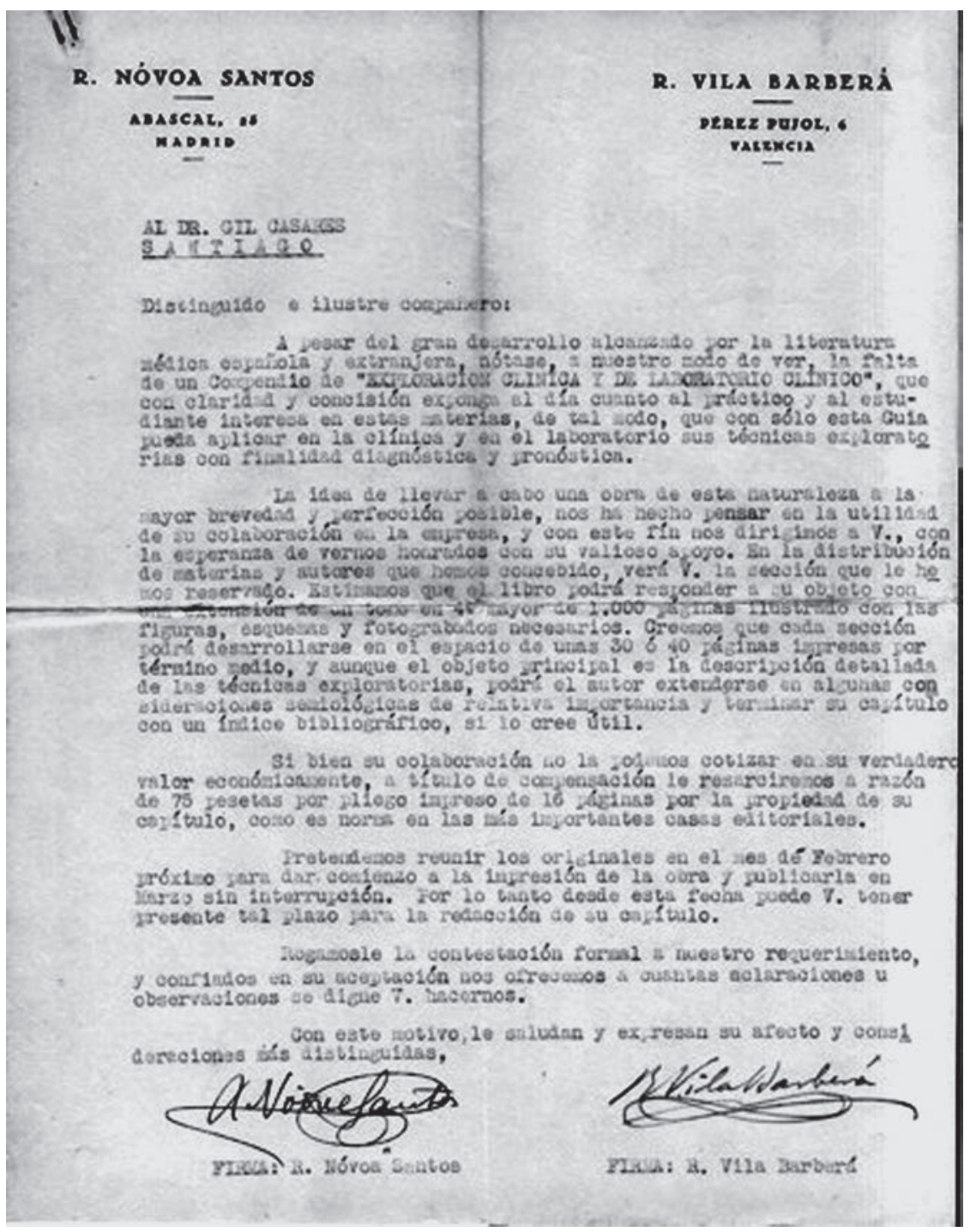

Fig. 2

a. Exploraciones Clínicas en general, bajo la dirección del Prof. Nóvoa Santos.

b. Exploraciones de Laboratorio, a cargo del Prof. D. Ramón Vila Barberá y su equipo.

c. Exploración Radiológica por el Dr. D. Vicente Carulla Riera. 


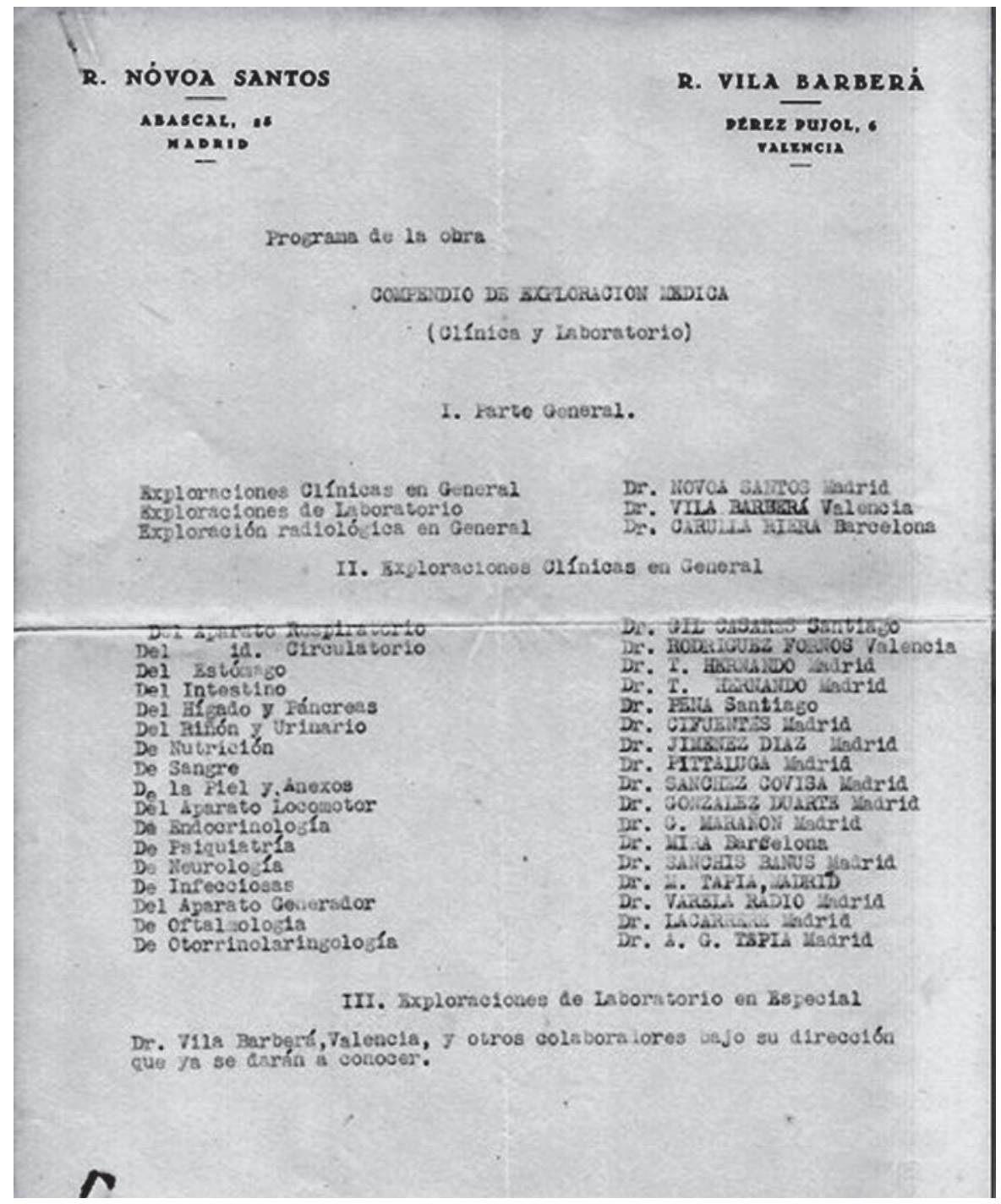

Fig. 3

\section{EL PROYECTO}

El proyecto de Compendio estaba pensado a lo grande, dadas las personalidades, casi todos destacados catedráticos, que fueron invitadas a colaborar en el mismo. Así, mientras las exploraciones especiales de laboratorio, se hacía cons- 
tar que, serían desarrolladas por Vila Barberá y "Otros colaboradores bajo su dirección que ya se darán a conocer”, la parte general que dirigía Nóvoa Santos la dividieron de la siguiente manera:

El apartado de Aparato Respiratorio se lo solicitaron a D. Miguel Gil Casares $^{5}$, el de Circulatorio al Dr. D. Fernando Rodríguez Fornos, catedrático de Clínica Médica, luego decano y rector de la Universidad de Valencia. Los capítulos de Estómago e Intestino a D. Teófilo Hernando, catedrático de Madrid, codirector con Marañón, desde 1916, del Manual de Medicina Interna que se ha venido conociendo como “El Hernando y Marañón”, ambicioso proyecto, el primero de su especie, hecho íntegramente por autores españoles. De Hígado y Páncreas quedaba encargado D. Pedro Pena, catedrático de Santiago, discípulo y sucesor de Nóvoa Santos, experto en el arte de la percusión. De Riñón y Urinario se ocuparía el Dr. D. Pedro Cifuentes y Díaz, director del Hospital de San Rafael de Madrid y decano del de la Princesa, presidente de la Sociedad Española de Urología y numerario de la Real Academia de Medicina.

La Nutrición quedaba adjudicada a D. Carlos Jiménez Díaz; el capítulo de Sangre a Gustavo Pittaluga, también catedrático, higienista y parasitólogo; el de Piel y anexos a D. José Sánchez Covisa, Catedrático, Académico de la Nacional de Medicina y Jefe de Servicio del Hospital de San Juan de Dios; el del Aparato locomotor a D. Plácido González Duarte, cirujano ortopédico y torácico; el de

\footnotetext{
5 Roberto Nóvoa Santos había sido alumno y compañero de claustro de D. Miguel (1871-1931) y el Dr. Vila también conocía a Gil Casares desde que, en 1918, el Dr. Gil Casares envió su trabajo sobre las aplicaciones del Palógrafo (del griego palós: palpitar), aparato de su invención: Un nuevo método registrador del pulso, al Instituto Médico Valenciano, al premio Dr. Faustino Roel, bajo el lema "Perseverancia", no siendo el trabajo admitido por motivos extracientíficos. Se le acusó de no ser el trabajo inédito por haber presentado el palógrafo en la Real Academia Nacional de Medicina. Esto lo criticó el Dr. Vila, pues según las bases del Premio Roel esa incompatibilidad no regía para los casos de trabajos o descubrimientos destacados.

Bajo los auspicios de el Prof. Vila Barberá, la revista Policlínica lo publica y, en su presentación, el propio profesor critica la actitud del Instituto Valenciano y elogia al Prof. Gil Casares, ensalzando su prestigio internacional afirmando que:

"Con sus estudios ha llegado no sólo a esclarecer los mecanismos fisiológicos y patológicos de las ondas de los registros cardíacos, sino que como los genios legítimos, ha creado, dotando a la ciencia de nuevos métodos de investigación, que van a revolucionar el instrumental de la Fisiología y la Clínica moderna".

Vid. Miguel Gil Casares, "Un nuevo método registrador del pulso", Policlínica: Revista de Medicina, Cirugía y Especialidades, núm. 65, (mayo 1918), pág. 36.

Nóvoa también pudo tener relación personal con Vila puesto que, además de ser titulares de la misma asignatura y cátedra, D. Roberto dio, en 1929, dos conferencias en Valencia a las que asistió Laín Entralgo, alumno de Medicina, de 21 años entonces, que quedó hondamente impresionado Vid. Pedro Laín Entralgo, Descargo de Conciencia (1930-1960), Barcelona, Edit. Barral, 1976, pág. 67, y Fernando Ponte Hernando, Roberto Nóvoa Santos (1885-1933) precursor del tratamiento de la diabetes con incretinas, Madrid, Ed. Novo Nordisk, d.1. 2009, págs. 68-69.
} 
Endocrinología a D. Gregorio Marañón; el de Psiquiatría al Prof. Mira de Barcelona; el de Neurología al Dr. Sanchís Banús, con el Dr. Mira, destacada figura de la Neurociencia clínica de la época, ya que, en lo que respecta a la Neurociencia experimental, aún vivía y trabajaba el maestro Cajal con su equipo. El de enfermedades Infecciosas a D. Manuel Tapia, tisiólogo, primer director del Hospital del Rey, discípulo de Madinaveitia y Marañón; y el de Aparato Generador (sic) a D. Manuel Varela Radío, catedrático de Obstetricia de la Universidad Central, antes de la de Santiago y, con Marañón, uno de los amigos que animaron a Nóvoa Santos a presentarse, en 1927, a la cátedra de Madrid.

De oftalmología se encargaría otro gallego, el joven Julio López Lacarrere, autor, en ese año, del famoso Libro-Atlas de Bio-microscopía de la córnea, iluminada con lámpara de doble hendidura. Finalmente, de Otorrinolaringología se haría cargo D. Antonio García Tapia, primer catedrático de O.R.L. por oposición, Académico de la Real de Medicina y médico de la Armada.

Como vemos, un elenco selecto y ambicioso en general.

También era un proyecto de categoría, desde el punto de vista formal, ya que los impulsores estimaban que se compondría de:

Unas mil páginas en cuarto mayor ilustrado con las figuras, esquemas y fotograbados necesarios.

Calculaban, asimismo, que cada sección podría constar de 30 a 40 páginas y,
Aunque el objeto principal es la descripción detallada de las técni- cas exploratorias, podrá el autor extenderse en algunas considera- ciones semiológicas de relativa importancia y terminar su capítulo con un índice bibliográfico si lo cree útil.

Desconocemos qué empresa editorial tenían o pensaban concertar, aunque podemos suponer que se tratase de la Editorial Saturnino Calleja, de cuya Sección de Monografías de Medicina y Biología eran codirectores: García Tapia, Marañón, Hernando y Sanchís Banús, y para la que Nóvoa estaba elaborando su libro Diabetes espuria y diabetes genuina, que se publicó en 1930. No obstante, la citada colección no estaba editada, como se pretendía en este compendio, "en cuarto mayor", o sea de 27 a 30 cms. sino en octavo, 18 cms. Debía tratarse de 
una edición no lujosa pues ofrecen a los autores 75 pesetas por pliego impreso de 16 páginas ${ }^{6}$ por la propiedad de su capítulo:

Como es norma en las más importantes casas editoriales, a título de compensación por no poder cotizar el verdadero valorar de las colaboraciones.

La carta viene sin fecha pero pretenden reunir los ejemplares "en febrero próximo para dar comienzo a la impresión de la obra y publicarla en marzo sin interrupción", lo que nos hace suponer que sea un texto escrito, aproximadamente, hacia el verano de 1929.

Decimos esto porque Nóvoa Santos se incorporó a la cátedra de Madrid a principios de 1928 e hizo un viaje de varios meses a Hispanoamérica en ese año y, quizás, pudo empezar a planteárselo hacia 1929. Luego, como veremos, el proyecto se frustró.

\section{Los gallegos y su papel en el proyecto}

De un total de 19 ponentes para 20 apartados, ya que D. Teófilo Hernando tenía a su cargo dos capítulos relativos a Estómago e Intestino, los gallegos suman un total de 5, lo que supone una proporción considerable, el 26\%. Éstos eran: el propio codirector D. Roberto Nóvoa Santos, D. Miguel Gil Casares, D. Pedro Pena Pérez, D. Manuel Varela Radío y el joven gallego-argentino D. Julio López Lacarrere. Respectivamente se hacían cargo de las temáticas siguientes: Exploraciones clínicas en general, Aparato Respiratorio, Hígado y Páncreas, Aparato Genital y Oftalmología.

\section{BREVES SEMBLANZAS}

\section{Roberto Nóvoa Santos (A Coruña 1885 - Santiago 1933)}

Médico y ensayista. Catedrático de Patología General, desde 1912 en la Universidad de Santiago de Compostela y, desde 1928, en Madrid. Se Licenció en Medicina con premio extraordinario en Santiago en 1907, doctorándose en Madrid en 1908 con la tesis: Alcance y sistematización de las cromorreacciones de los albuminoides. Comenzó como profesor auxiliar de Patología General en esa Facultad en 1911, año en que fue a estudiar Fisiología y Patología a Fran-

\footnotetext{
${ }_{6}$ Obra en nuestro poder otra carta en la que, diez años antes, en 1919, el Prof. Fidel Fernández Martínez para su monumental Tratado iberoamericano de Medicina Interna ofrece 150 pts. por la misma extensión de 16 páginas, en nombre de la Editorial Plus Ultra, a D. Miguel Gil Casares.
} 
cia, Austria y Alemania, con una bolsa de la J.A.E. Como consecuencia de esta estancia que continuó en 1912, siendo ya catedrático, publicó seis trabajos científicos notables.

Eminente clínico e investigador, son especialmente destacables sus investigaciones sobre la diabetes.

Según la última revisión, de J. J. Fernández Teijeiro y F. Ponte Hernando (en prensa), dejó 265 trabajos publicados. Introductor en España de las corrientes fisiopatológicas, siguiendo a Ludolf von Krehl, fue, según Rof Carballo, uno de los más tempranos divulgadores en España de la obra de Freud. Publicó numerosos trabajos sobre su especialidad, así como sobre Psicología, Estética y Antropología médica. Fue precursor del tratamiento de la diabetes con incretinas, con sus investigaciones desde $1924^{7}$.

Excelente escritor y ensayista, con influencias de William James ${ }^{8}$, alcanza cotas de notable profundidad y penetración psicológica y filosófica en estudios como: La inmortalidad y los orígenes del sexo (1931); La mujer, nuestro sexto sentido y otros esbozos (1929) y, en particular, a nuestro juicio, en El Instinto de la muerte o la Patografía de Santa Teresa de Jesús (ambos de 1932).

\section{Miguel Gil Casares (1871-1931)}

Nacido en Santiago de Compostela, de familia que dio ilustres intelectuales y científicos, como su abuelo, D. Antonio Casares Rodríguez, rector de la Universidad de Santiago; su tío abuelo, D. Maximino Teijeiro, también rector; su padre, D. Ramón Gil Villanueva, catedrático y decano de la Facultad de Ciencias; su tío, D. Jacobo Gil Villanueva, rector y destacado civilista; y sus tíos-primos, D. José Casares Gil, presidente de las Reales Academias de Farmacia y de Ciencias, y D. Antonio Casares Gil, Coronel médico y destacado botánico, a nivel internacional, especialista en briofitas ${ }^{9}$.

\footnotetext{
7 Vid. Fernando Ponte Hernando, La diabetes mellitus en la época y en la obra de Roberto Nóvoa Santos (1885-1933): un análisis histórico-médico, tesis doctoral [en línea], disponible en <http://hdl.handle.net/2183/8264> [Consulta: 30/04/2014].

8 Juan José Fernández TeiJeiro, "Ciencia, espiritualismo y religión: entre Nóvoa Santos y William James", Revista de Historia de la Psicología, vol. 34, núm. 4, (diciembre 2013), págs. 47-66.

9 Antonio, a la vez que médico militar, fue uno de los más afamados botánicos de España, tras haberse especializado en esta materia en los mejores laboratorios europeos, en contacto con sabios como Goebel o la escuela de su maestro Julius Sachs. Se le considera el primer briólogo de España.

Fue profesor de los cursos de botánica que la Junta de Ampliación de Estudios organizaba en el Museo Nacional, donando de su bolsillo diverso aparataje. vid. Residencia DE EstUdiAnTES, Archivo de la JAE [en línea], disponible en <http://archivojae.edaddeplata.org/jae_app/> [Consulta: 30/04/2014].

Las briofitas son un grupo de plantas verdes pequeñas muy importante en la evolución del reino vegetal. Había nacido en 1871 el mismo año que Miguel, murió en 1929, a los 58 años, con el grado 
D. Miguel fue catedrático de Enfermedades de la Infancia en Santiago de 1897 a 1901 y desde esa fecha a su muerte, en 1931, de Patología y Clínica Médicas. Senador por la Universidad de Santiago, autor de numerosas publicaciones y de algunos inventos, como su modificación sobre el aparato de neumotórax artificial para el tratamiento por colapsoterapia de las cavernas tuberculosas, y sobre todo el palógrafo, del griego palós, palpitar, instrumento precursor del fonocardiógrafo.

Este último invento lo patentó en España y Estados Unidos y le dio fama internacional, presentándolo en Madrid, París, Londres, Berlín, Nueva York y La Habana, siendo reconocido como uno de los inventos del año 1924 por la Sociedad de Naciones. Destacado especialista en Neumología y Tisiología, así como en Cardiología, tradujo diversos libros del alemán como los de Boas ${ }^{10}$ y Oswaldo Vierordt ${ }^{11}$, así como el Tratado de Medicina Interna de von Mering —en el que incluyó un capítulo propio sobre Lepra- dirigido por Krehl, quién, a su vez, prologó su obra sobre el palógrafo. Fue además fundador y primer director del gabinete radiológico de la Facultad de Medicina y el Hospital Clínico, el segundo de Galicia.

\section{Pedro Pena Pérez}

Colaborador y discípulo de Nóvoa Santos, ganó la cátedra de Patología General de Cádiz, que ocupó brevísimamente, por trasladarse a la de Santiago al quedar vacante la misma, por pasar su maestro a la de Madrid. En 1936 se traslada a la de Patología y Clínica Médicas, también en Santiago.

Había estudiado Fisiología y Patología de la nutrición y de las glándulas endocrinas en Zürich y Basilea ${ }^{12}$; con destacados científicos como Stähelin, Química fisiológica con G. V. Bumge y Secreciones Internas y Nutrición con R. Metzner en el verano de 1918 e invierno de 1918-1919. ${ }^{13}$ Interrumpió su pensión de mayo de 1919 a enero de 1920, luego continuó en Berna con Sähli con la beca

\footnotetext{
al menos temporal de General Subinspector Médico. (equivalente en las Armas a General de Brigada o Contralmirante). Archivo Histórico Militar de Segovia. Expte personal. N. del A.

${ }^{10}$ Ismar BoAs, Diagnóstico y Tratamiento de las enfermedades del estómago según el estado actual de la Ciencia, 2, vol. Madrid, 1902, Rev. Méd. y Cir. Prácticas, traducción: Rafael del Valle y Miguel Gil Casares.

${ }^{11}$ Oswaldo Vierordt, Diagnóstico de las enfermedades internas fundado en los actuales métodos de exploración: manual para estudiantes y médicos, Santiago de Compostela, El Eco de Santiago, 1907.

${ }^{12}$ Residencia de Estudiantes, Archivo de la JAE [en línea], disponible en <http://archivojae. edaddeplata.org/jae_app/ > [Consulta: 30/04/2014].

${ }^{13}$ Envió entonces a la Junta el trabajo: El cambio material de la colina.
} 
de la $\mathrm{JAE}^{14}$, y fue posteriormente becado por la Universidad de Santiago para estudiar, en París, los nuevos métodos de exploración clínica.

Autor de numerosos trabajos sobre todo de Aparato Digestivo y Nutrición, fue secretario y decano de la Facultad de Medicina, vicerrector y nombrado rector por las autoridades militares, tras el Alzamiento de 1936, siendo cesado tres meses después.

\section{Manuel Varela Radío (1873-1962) $)^{15}$}

Nacido en Pontevedra en 1873, hijo de Teodoro Varela de la Iglesia, catedrático de Matemáticas del Instituto de aquella ciudad, y de Salomé Radío de la Vega ${ }^{16}$, que murió cuando el pequeño Manuel tenía 10 años. Era, por tanto, sobrino del célebre catedrático de Fisiología de Santiago, D. Ramón Varela de la Iglesia ${ }^{17}$.

Alcanzó gran prestigio:

Fue uno de los más famosos ginecólogos de España. Era tan admirable operador como insuperable maestro. Dominaba sobre todo las técnicas vaginales, en particular las de las fístulas, habiendo llegado a tener en este tipo de intervenciones una de las mejores estadísticas del mundo. Formó gran número de magníficos especialistas. Uno de sus primeros discípulos fue Alejandro Otero, que obtuvo la cátedra de Ginecología de Granada y fue Rector de aquella Universidad, llegando a tener una amplia reputación internacional $^{18}$.

Ganó la plaza de profesor auxiliar de Santiago en oposición celebrada en Valladolid, por unanimidad, tras haberse retirado el resto de los opositores.

\footnotetext{
${ }^{14}$ Donde preparó un trabajo sobre el método yodométrico del profesor Sahli que apareció en 1920 en Archivos Españoles de Enfermedades del Aparato Digestivo y de la Nutrición.

${ }^{15}$ Extractado de Fernando Ponte Hernando, Dr. Ángel Baltar Cortés (1868-1934) Maestro de Cirujanos, A Coruña, Eds. Inéditor-Grupo Towers, 2011, págs. 67-69.

${ }^{16}$ Manuel Varela UÑa, De Memoria: A fuerza de Tiempo, Madrid, Ed. Taurus, 2009, pág.27.

${ }^{17}$ Vid. Juan José Fernández Teijeiro, Ramón Varela de la Iglesia (1845-1922) Positivismo e Histología en Fonseca, [CD ROM], Santiago de Compostela, Universidade de Santiago de Compostela, Servizo de Publicacións e Intercambio Científico, d.1. 2008, págs. 56-57.

${ }^{18}$ Ramón Baltar Domínguez, La escuela médica compostelana: $21^{a}$ exposición con motivo del centenario de la muerte del Dr. Varela de Montes, Santiago, Instituto Padre Sarmiento de Estudios Gallegos, 1968, págs. 25-26.
} 
Entre su escasa obra escrita ${ }^{19}$, norma común a todos los miembros de la Escuela Médica Compostelana, hay que señalar la traducción de la segunda edición alemana de la obra de su amigo, el Prof. Heinrich Martius, de Göttingen: Operaciones ginecológicas y sus fundamentos anatomotopográficos ${ }^{20}$.

Publicó en diversas revistas especializadas artículos entre los que cabe señalar: "La marsupialización como medio de tratamiento de algunos tumores quísticos", siendo, al menos nominalmente, colaborador permanente de la Revista General de Medicina y Cirugía.

Fue uno de los introductores en España de las técnicas de Cirugía vía vaginal y pionero en algunas técnicas como la panhisterectomía de Wertheim y Schauta, tanto por vía abdominal como vaginal. Resultó muy celebrado su trabajo: Formación de una vagina artificial a expensas de un trozo de intestino delgado. Tuvo un importante papel en la introducción de la radioterapia moderna en el primer Sanatorio Quirúrgico de Galicia (1908) el Sanatorio Baltar-Varela, fundado por él mismo y por D. Ángel Baltar Cortés.

En Alemania, Francia y Austria, se formó con Bumm, Doderlein, Leopold, Diesen, Pick y O. Hertig; Schauta y Olhausen fueron sus mentores ${ }^{21}$.

\section{Julio López Lacarrere (1895-1964)}

Joven y prestigioso oftalmólogo gallego-argentino que: A la edad de un alumno es ya un admirado maestro en oftalmología ${ }^{22}$. Había nacido en Buenos Aires, hijo del industrial español D. Julio López Bailly y Marcelina Isabel Lacarrere, uruguaya. Como curiosidad decir que el padre fue el promotor de las Casas Bailly de San Pedro de Nós, A Coruña. Fueron construidas entre 1920 y 1924 por los arquitectos Antonio Tenreiro y Peregrín Estellés, en marcado estilo modernista europeo con bellos mosaicos, lucernario y jardines. Los Bailly eran una familia gallega acomodada, descendientes de un soldado de Napoleón, que poseía varias fábricas en Argentina ${ }^{23}$. En los años 50, el doctor D. Julio López

\footnotetext{
${ }^{19}$ Su tesis doctoral versó sobre Formas clínicas de afasia, un tema extraño para lo que sería toda su trayectoria posterior.

${ }^{20}$ Fue traducida del alemán por el Prof. M. Varela Radio y posteriormente puesta al día de la $7^{\mathrm{a}}$ Ed. Alemana por su hijo el Dr. Varela Uña: Heinrich MARTIUs, Operaciones ginecológicas y sus fundamentos anatomotopográficos, traducción del alemán por el Prof. M. Varela Radío; puesta al día, según la séptima edición alemana, por el Dr. M. Varela Uña, Barcelona, Labor, 1957.

${ }^{21}$ Boletín Oficial del Colegio Practicantes de Santiago Compostela, Año 2, (Enero 1929), pág. 3-4.

${ }^{22}$ Enrique Hervada García Sampedro, "Epílogo" en Andrés Martínez Morás, Semblanzas raciales: primera serie, Madrid, Espasa-Calpe, 1930, pág. 196.

${ }^{23}$ Vid. Blog Curiosos Incompletos, La casa Bailly. Mansión abandonada [en línea], disponible en <http://curiososincompletos.wordpress.com/2011/09/24/la-casa-bailly-mansion-abandonada> [Consulta: 30/04/2014].
} 
Lacarrere está en Madrid, al menos un tiempo, como Ministro Consejero de la Embajada Argentina ${ }^{24}$.

Cursa Alemán en la escuela de Náutica de La Coruña, ciudad en la que su hermano Eduardo, tres años menor, sigue el bachillerato comenzado en el Colegio Nacional Mariano Moreno de Buenos Aires, y que terminará en el Instituto de Santiago. De 1914 a 1918 estudia Medicina en la Facultad de la Universidad de Santiago ${ }^{25}$, al igual que Eduardo ${ }^{26}$, que lo hace de 1914 a 1919. Ambos terminarán la carrera en Madrid. Julio fue profesor del Hospital del Niño Jesús ${ }^{27}$ y destacaba en la ciencia y en la sociedad madrileña, siendo socio del Ateneo, y habiendo marcado un hito con su Libro-Atlas de Bio-microscopía de la córnea, iluminada con lámpara de doble hendidura y sus inventos oftalmológicos:

A partir de la presentación en Sevilla de «La Potenza», que permitía por su fácil manejo el estudio de fondo de ojo, incluso en enfermos con poca colaboración, fueron varios los aparatos de exploración y tratamiento diseñados por él. Destacando entre otros, el «Fotocampímetro Registrador», el «Electrodiafaco» y el «Myocampter», modificado sobre un modelo de Barraquer ${ }^{28}$.

Ejerció, con eficacia, la secretaría de las cruciales Jornadas Médicas gallegas, de 1929, en las que se volcaron todos los grandes de la Medicina madrileña naturales de Galicia: D. Jacobo López Elizagaray, que las presidió; Varela y Varela y Gómez Ulla, médicos militares y de Cámara de Alfonso XIII; y catedráticos como Nóvoa Santos, Varela Radío, o figuras de la cirugía como Goyanes Capdevila, estando todos ellos en el Comité de Honor y Julio en el organizador. También fue Secretario General del XIV Congreso Internacional de Oftalmología en Madrid, en 1933.

Enviudó en febrero de 1932 de Manuela López Olmos, posteriormente retornó a la República Argentina donde contrajo nuevas nupcias, continuó su labor,

\footnotetext{
${ }^{24}$ Periodico ABC, viernes 21 de febrero de 1958, pág. 19.

${ }^{25}$ Archivo Historico Universitario de Santiago (en adelante sólo AHUS), Leg, 721, Expte. 10.

${ }^{26}$ AHUS, Leg, 721, Expte, 9.

${ }^{27}$ Periodico ABC, viernes 21 de febrero de 1958, pág. 19.

${ }^{28}$ C. Jiménez, D. Puertas, A. Cortés, P. Navarro, "El Hospital Niño Jesús de Madrid en la génesis de la Sociedad Española de Estrabología", Acta Estrabológica, [en línea], disponible en <http:// www.oftalmo.com/estrabologia/rev-96/96-02.htm> [Consulta: 30/04/2014]. Con poco rigor dicen que la guerra de 1914 le obligó a quedarse en España, al llegar de Argentina, cuando tenía intención de viajar por Europa. A raíz de lo expuesto sobre sus padres y hermano, y sus estudios en Santiago y consulta en Madrid, esto parece claramente inexacto. Además no figura entre los becados por la JAE, quizás por no ser español.
} 
donde fallecería en 1964. Tanto él como su hermano Eduardo figuran como Doctores por la Universidad Nacional de La Plata en 1937, probablemente por haber tenido que renovar el título español allí en unas condiciones durísimas para los titulados en España, como era habitual.

\section{Resultado Del Proyecto}

El que el proyecto no siguiese adelante tal y como estaba previsto y sí una versión netamente valenciana del mismo, a cargo de Vila Barberá, con colaboradores como López Ibor y Nicasio Belloch, fue debido a que, en breves meses, se producen los siguientes fallecimientos: el 11 de abril de 1931 el de Gil Casares, precedido de larga enfermedad; el de Sanchís Banús, en julio de 1932; y el del propio Nóvoa Santos, en diciembre de 1933, también precedido de graves padecimientos, tras ser intervenido por una neoplasia gástrica que resultó inoperable por D. Mariano Gómez Ulla ${ }^{29}$.

\section{VILA TOMA LAS RIENDAS}

El Dr. D. Ramón Vila Barbera (Fig.4), probablemente obligado por este estado de cosas, decide reorientar la idea y publica como autor principal la obra Medicina Exploratoria. Clínica y Laboratorio. Ignoramos porqué decidió hacer una obra mucho más voluminosa de la prevista en un principio.

La intención inicial es publicar 5 ó 6 volúmenes, 2 ó 3 por año. Finalmente sólo salen a la luz 4 de ellos. El quinto y último no se publicó, aunque parece que sí estaba totalmente redactado, tal y como refleja Vila en el prólogo del $4^{\circ}$ volumen que publicó en 1941, año en que se produce su fallecimiento en el balneario de Liérganes (Cantabria), donde era director médico durante los meses de la temporada de baños.

Vila inicia el primer tomo explicando las razones de la decisión de publicar el mismo bajo el lema: Utilidad, claridad y actualidad. Expone que el alumno y el médico precisan tener un instrumento que facilite el trabajo seguro y firme a la cabecera del enfermo, en una extensión completa, incluyendo datos de laboratorio, pero no enciclopédica como algunos de los manuales preexistentes ni tan limitada como en otros, subrayando la labor orientadora en lo teórico y demostradora en lo práctico del catedrático y sus ayudantes.

\footnotetext{
${ }^{29}$ Mariano Gómez Ulla (1877-1945) nacido en Compostela, fue gentilhombre de cámara de Alfonso XIII, luego en la postguerra, General Inspector de Sanidad Militar, Presidente del Consejo General de Colegios Médicos de España y numerario de la Real Academia Nacional de Medicina. Durante su cruel cautiverio (1936-1938) en zona republicana Los hermanos Lacarrere fueron impulsores de su liberación entre sabios como Houssay a nivel internacional.
} 


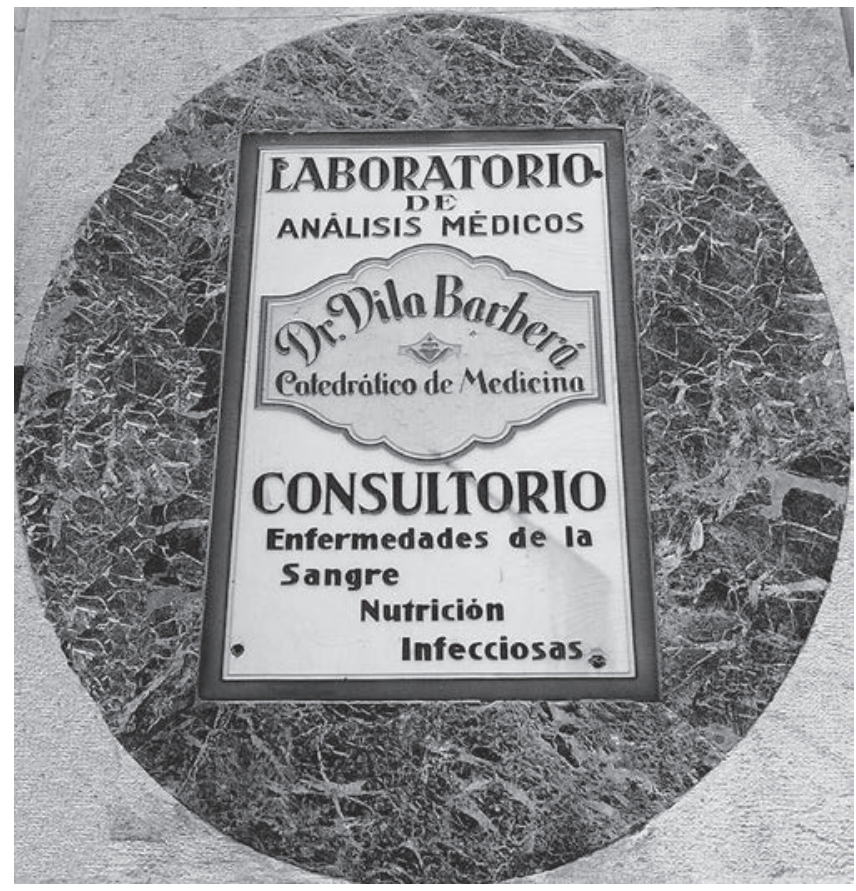

Fig. 4

El primer volumen (Fig. 5) aparece publicado en el año 1932; en él, aparte del dibujante e ilustrador, sólo colabora el Dr. Tomás Zumalacárregui Calvo, profesor de la Facultad de Medicina de Valencia y discípulo del Dr. Vila, con "La acción dinámica específica y el cociente respiratorio". El segundo, dedicado al Sistema Nervioso, se publica en 1934 y se retrasa su publicación, tal y como hace constar el Dr. Vila Barbera en el prólogo, por motivos ajenos a su voluntad. En este tomo colaboran:

- Dr. D. Rafael Climent Pardo, médico del Cuerpo de Beneficencia y Sanidad (Síndromes cerebrales espéciales: trastornos del lenguaje, sueño e insomnio. Psiquismo y exploración psiquiátrica).

- Prof. Dr. D. Jesús Basterra Santacruz, catedrático de Oftalmología desde 1932, (Campo visual, sentido cromático y agudeza visual).

- Prof. Dr. D. Rafael Bartual Vicens que, en 1945, sería catedrático de ORL (Exploración vestíbulo-acústica).

- Dr. D. Miguel Verdeguer Pons, médico decano del Cuerpo Municipal de Beneficencia y Sanidad (Exploración de las funciones olfatoria, gustativa, deglución y laríngea). 


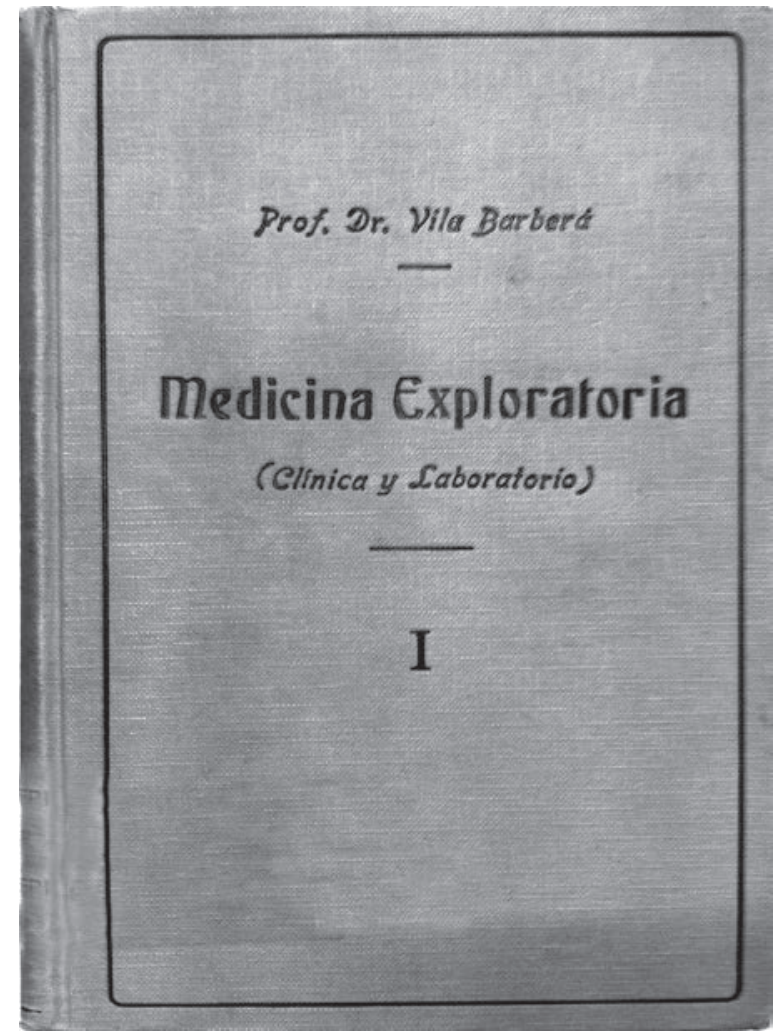

Fig. 5

- Prof. Dr. D. Juan José López Ibor, catedrático de Medicina Legal, luego de Psiquiatría (Exploración eléctrica).

El volumen tercero lo dedica a la exploración respiratoria y cardíaca. Se publicó en 1936 y colaboraron: el Prof. Dr. D. José Campos Igual, catedrático interino de Otorrinolaringología y el Dr. D. José Campos Peña, profesor auxiliar de la Facultad de Medicina, que elaboraron el capítulo de exploración rinofaríngea y traqueo-broncoscopia. A cargo del Dr. D. Antonio Vila López, ex ayudante del Dr. Vila Barberá en su servicio clínico, y médico del cuerpo de Sanidad Militar, estuvo el capítulo de electrocardiografía clínica y exploración cardiovascular.

El cuarto volumen sale publicado en 1941, tras la guerra civil española, y en plena segunda guerra mundial. En el prólogo comenta los problemas que han tenido para conseguir papel. En este tomo destaca como colaborador el Dr. D. Nicasio Benlloch Giner, urólogo, con cateterismos y endoscopias en vías urinarias. Todos los volúmenes fueron impresos en los talleres de José Olmo, en Valencia. 


\section{CONCLUSIONES}

1. El que hubiera sido el primer libro de semiología y técnica exploratoria, tanto clínica como de laboratorio y radiología, íntegramente realizado por los profesionales más importantes de la medicina española que se codeaban con los sabios internacionales, se frustró, probablemente por la sucesión de fallecimientos tempranos de varios de los autores.

2. El papel de los facultativos gallegos es sumamente destacado en cantidad al constituir casi el 30\% de los mismos y en calidad, dado el alto nivel de las importantes figuras invitadas a participar.

3. El proyecto alternativo, impulsado por el Prof. D. Ramón Vila Barberá, resultó un producto sumamente digno, bastante extenso y hecho por notables personajes de la medicina, aunque; más restringido al área de Valencia; resultando inconcluso al no publicarse su $5^{\circ}$ y último volumen por el fallecimiento de su director.

\section{BIBLIOGRAFÍA}

Baltar Domínguez, Ramón, La escuela médica compostelana: $21^{a}$ exposición con motivo del centenario de la muerte del Dr. Varela de Montes, Santiago, Instituto Padre Sarmiento de Estudios Gallegos, 1968.

Blog Curiosos Incompletos, La casa Bailly. Mansión abandonada [en línea], disponible en $<$ http://curiososincompletos.wordpress.com/2011/09/24/la-casa-bailly-mansion-abandonada> [Consulta: 30/04/2014].

Boas, Ismar, Diagnóstico y Tratamiento de las enfermedades del estómago según el estado actual de la Ciencia, vol. 2,Madrid, 1902.

Boletín Oficial del Colegio Practicantes de Santiago Compostela, Año 2, (Enero 1929), págs. 3-4.

Campos Fillol, Rafael, Crónica de la Facultad de Medicina de Valencia (De 1866 a 1946), Discurso de recepción del académico electo Prof. Rafael Campos Fillol, Valencia, Real Academia de Medicina de Valencia, 1955.

Fernández Teijeiro, Juan José, Roberto Nóvoa Santos: La inmortalidad, dolor y saudade, Sada (A Coruña), Eds. Do Castro, 2003, págs. 71-89.

Fernández Teijeiro, Juan José, Ramón Varela de la Iglesia (1845-1922) Positivismo e Histología en Fonseca, [CD ROM], Santiago de Compostela, Universidade de Santiago de Compostela, Servizo de Publicacións e Intercambio Científico, d.1. 2008.

Fernández Teijeiro, Juan José y Ponte Hernando, Fernando, “Tras las huellas de un patólogo: Roberto Nóvoa Santos (1885-1933)", El Médico: profesión y humanidades, núm. 1089 (1-15 feb 2009), págs. 42-50.

Fernández Teijeiro, Juan José, “Ciencia, espiritualismo y religión: entre Nóvoa Santos y William James", Revista de Historia de la Psicología, vol. 34, núm. 4 (diciembre 2013), págs. 47-66. 
Gil Casares, Miguel, "Un nuevo método registrador del pulso”, Policlínica, Revista de Medicina, Cirugía y Especialidades, núm. 65, (Mayo 1918), págs. 36-45.

Hervada García Sampedro, Enrique, "Epílogo”, en Andrés Martínez Morás, Semblanzas raciales: primera serie, Madrid, Espasa-Calpe, 1930, págs.191-197.

Jiménez, C.; Puertas, D.; Cortés, A.; Navarro, P., "El Hospital Niño Jesús de Madrid en la génesis de la Sociedad Española de Estrabología", Acta Estrabológica, [en línea], disponible en <http://www.oftalmo.com/estrabologia/rev-96/96-02.htm> [Consulta: 30/04/2014].

Laín Entralgo, Pedro, Descargo de Conciencia (1930-1960), Barcelona, Ed. Barral, 1976.

López Piñero, José María, Historia de la Medicina Valenciana, tomo III, Valencia, Ed. Vicent García, 1992.

Martínez Morás, Andrés, Semblanzas raciales: primera serie, Madrid, Espasa-Calpe, 1930.

Martius, Heinrich, Operaciones ginecológicas y sus fundamentos anatomotopográficos, traducción del alemán por el Prof. M. Varela Radío; puesta al día, según la séptima edición alemana, por el Dr. M. Varela Uña, Barcelona, Labor, 1957.

Noguer Molins, Luis, Exploración Clínica, Barcelona, Ed. Científico-Médica, 1916.

Ponte Hernando, Fernando, Roberto Nóvoa Santos (1885-1933) precursor del tratamiento de la diabetes con incretinas, Madrid, Ed. Novonordisk, d.1. 2009.

Ponte Hernando, Fernando y Posse Vidal, Luis, "Miguel Gil Casares: Señor de la Medicina", Cuadernos de Atención Primaria, vol. 17, núm. 1, (2010), págs. 81-83.

Ponte Hernando, Fernando, La diabetes mellitus en la época y en la obra de Roberto Nóvoa Santos (1885-1933): un análisis histórico-médico, tesis doctoral [en línea], disponible en $<$ http://hdl.handle.net/2183/8264> [Consulta: 30/04/2014].

Ponte Hernando, Fernando, Dr. Ángel Baltar Cortés (1868-1934) Maestro de Cirujanos, A Coruña, Eds. Inéditor-Grupo Towers, 2011, págs. 67-69.

Residencia de Estudiantes, Archivo de la JAE [en línea], disponible en <http://archivojae. edaddeplata.org/jae_app/> [Consulta: 30/04/2014].

Seifert, Otto y Müller, Friedrich, Guía del diagnóstico médico, Madrid, Establecimiento Tipográfico de Enrique Teodoro, 1887.

Varela Uña, Manuel, De Memoria: A fuerza de Tiempo, Madrid, Ed. Taurus, 2009.

Vierordt, Oswaldo, Diagnóstico de las enfermedades internas fundado en los actuales métodos de exploración: manual para estudiantes y médicos, Santiago de Compostela, El Eco de Santiago, 1907.

Vila Barberá, Ramón, Medicina exploratoria: (clínica y laboratorio), Valencia, Imprenta de José Olmos, 1932-1941. 